\title{
Unmet Dental Needs in Rural Primary Care: A Clinic-, Community-, and Practice-based Research Network Collaborative
}

\author{
Melinda M. Davis, PhD, MA, Thomas J. Hilton, MS, DMD, Sean Benson, DDS, \\ Jon Schott, MD, Alan Howard, MS, Paul McGinnis, MPA, and Lyle Fagnan, MD
}

Background: Oral health is an essential component of general health and well-being, yet barriers to the access of dental care and unmet needs are pronounced, particularly in rural areas. Despite associations with systemic health, few studies have assessed unmet dental needs across the lifespan as they present in primary care. This study describes the prevalence of oral health conditions and unmet dental needs among patients presenting for routine care in a rural oregon family medicine practice.

Methods: Eight primary care clinicians were trained to conduct basic oral health screenings for 7 dental conditions associated with International Statistical Classification of Diseases and Related Health Problems 9-Clinical Modification codes. During the 6-week study period, patients older than 12 months of age who presented to the practice for a regularly scheduled appointment received the screening and completed a brief dental access survey.

Results: Of 1655 eligible patients, $40.7 \%(n=674)$ received the screening and $66.9 \%(n=1108)$ completed the survey. Half of the patients who were screened $(46.0 \%, n=310)$ had oral health conditions detected, including partial edentulism (24.5\%), dental caries (12.9\%), complete edentulism (9.9\%), and cracked teeth (8.9\%). Twenty-eight percent of the patients reported experiencing unmet dental needs. Patients with dental insurance were significantly more likely to report better oral and general health outcomes as compared with those who had no insurance or health insurance only.

Conclusions: Oral health diseases and unmet dental needs presented substantially in patients with ages ranging across the lifespan from one rural primary care practice. Primary care settings may present opportune environments for reaching patients who are unable to obtain regular dental care. (J Am Board Fam Med 2010;23:514-522.)

Keywords: Practice-based Research, PBRN, Oral Health, Rural Health, Community-based Participatory Research

The Surgeon General's 2000 Report notes that oral health is an essential component of the general health and well-being of all Americans. ${ }^{1}$ Oral health influences productivity, quality of life, and systemic health. ${ }^{2,3}$ Dental conditions such as periodontal (gum) disease, a source of chronic infection and inflammation in the oral cavity, have been

This article was externally peer reviewed.

Submitted 14 April 2009; revised 13 August 2009; accepted 20 August 2009.

From the Oregon Rural Practice-based Research Network (MMD, LF, PM) and the School of Dentistry (TJH), Oregon Health and Science University, Portland; and the Department of Family Medicine (LF), Oregon Clinical and Translational Science Institute (LF, PM), Portland; private practice, Baker City (JS, SB); and Academic Computing Services, The University of Vermont, Burlington (AH).

Funding: This research was supported by a Clinical and Translational Science Award to Oregon Health and Sciences University (National Institute of Health/National Center for Research Resources grant no. 1U1 RR02414-01). associated with atherosclerosis, diabetes, adverse pregnancy outcomes, and increased risk of myocardial infarction and stroke. ${ }^{1,4,5}$ This may be a result of common pathophysiologic pathways that are involved in inflammation and altered host response. ${ }^{6}$ Tooth decay is the most common chronic disease in childhood, occurring 5 to 8 times more often than asthma. ${ }^{7,8}$

Many oral health conditions can be detected early and are preventable with appropriate care. ${ }^{9}$ However, researchers have identified dental care as the most frequently reported unmet health need in national studies of both adult and youth popula-

Conflict of interest: none declared.

Corresponding author: Melinda M. Davis, PhD, MA, Oregon Rural Practice-based Research Network, 3181 SW Sam Jackson Park Road, Portland, OR 97239 (E-mail: davismel@ohsu.edu). 
tions. ${ }^{9,10}$ Although annual dental examinations are an important form of preventive care, approximately $44 \%$ of Americans lack dental insurance and therefore may experience difficulties accessing nonemergency dental services. ${ }^{9,11}$ Researchers have also documented disparities in the access and use of dental care among rural populations. ${ }^{12-16}$ Compared with their urban counterparts, rural residents of all ages are more likely to have unmet dental needs and to report that their last dental visit was because something was "bothering or hurting."13

Objectives of Healthy People 2010 indicate a need to increase the proportion of adults who use the oral health care system annually and to increase the proportion of low-income children and adolescents who receive preventive dental services. ${ }^{3}$ The availability of dental providers and primary care is associated with healthier teeth. ${ }^{17}$ Because of the linkage between oral health and chronic illness, experts encourage collaborative approaches between dental providers, public health, and other health care professionals to address oral health disparities. $^{18-22}$

Many consider primary medical care as a venue for reaching children who do not traditionally make dental visits. ${ }^{23,24}$ Primary care medical practices in rural Oregon communities report few resources for patients who present with dental health needs. ${ }^{25}$ Rural clinicians in our primary care research network (the Oregon Rural Practice-based Research Network) wanted to confirm their impression that many patients present to their practices with dental health issues. In addition, local community leaders in medicine, dentistry, and public health sought baseline data regarding the prevalence of unmet dental needs to determine how to best address this local health concern.

We undertook this cross-sectional study to quantify unmet dental needs and oral health conditions in a rural primary care practice. The study emerged as a community-based participatory research project involving members of the county Community Health Improvement Partnership, ${ }^{26}$ local medical and dental providers, and representatives from 2 practice based research networks (PBRNs) at Oregon Health and Science University (OHSU). One PBRN focused on oral health (Practice-based Research in Oral Health) and the second on rural primary care (Oregon Rural Practicebased Research Network). Though an increasing body of literature is exploring pediatric partner- ships to address childhood oral health, there is little work that addresses the prevalence of oral health conditions among all patients who present to a family medicine practice for routine medical care. ${ }^{22,27-30}$ We hypothesized that there would be a high prevalence of dental conditions and unmet dental needs as identified by family physicians in routine primary care practice. We also hypothesized that the conditions would be distributed in predictable ways, with caries most common among children and varying degrees of edentulism most common among the elderly.

\section{Methods}

\section{Selection and Description of Participants}

We collected data in a private family medicine practice located in a frontier county in rural eastern Oregon with 13,862 residents. ${ }^{31}$ Patients older than 12 months of age who presented to the practice for a regularly scheduled visit during the 6-week study period of January 2, 2008, to February 13, 2008, were eligible for participation.

Eight primary care providers (4 physicians, 1 physician assistant, and 3 family nurse practitioners) staffed the clinic and cared for more than $50 \%$ of patients in the local service area. ${ }^{32}$ The clinic's patient panel was 10,$977 ; 59.7 \%$ of the patients were women and more than $99 \%$ were white. Two percent of the patients were younger than 1 year of age, $19 \%$ were between 1 to 14 years old, and $21 \%$ were older than 65 years old. The clinic accepted multiple payment sources, with $51 \%$ of revenue in 2008 from private insurance, $30 \%$ from Medicaid, 15\% from Medicare, 3\% from self-pay, and $1 \%$ from Tricare/Champus.

\section{Data Collection}

Clinic providers conducted a basic oral health screening and patients or their caregivers completed a self-report survey about dental needs and access to care. There were no extra costs to patients or third-party payers for study participation and neither study participants nor clinic staff received financial compensation. The authors developed study tools and methodologies in conjunction with clinic staff and providers. The OHSU Institutional Review Board approved this study.

\section{Oral Health Screening}

Using a mix of diagnostic photographs and didactic consultation, the local dentist (SB), in collaboration 
with faculty at the OHSU School of Dentistry (TH), trained participating clinicians. Instructors taught the primary care providers to identify 7 basic oral health conditions associated with International Statistical Classification of Diseases and Related Health Problems 9-Clinical Modification diagnosis codes, including dental caries (cavities, 521.00); broken/cracked teeth (521.81); abscess (522.5); complete edentulism (no teeth, 525.40); partial edentulism (missing teeth, 525.50); unspecified disorder of teeth (525.9); and no conditions observed (V70.7). Conditions were based on the Decayed, Missing, and Filled Teeth Index (DMFT), a tool oral health professionals use for recording tooth surface conditions in epidemiologic studies, and from dental conditions commonly observed during visits to hospital emergency rooms. ${ }^{33,34}$ The dental mentors were available for onsite feedback and guidance on the first day of the intervention and via telephone consult throughout the study period.

The project leaders asked clinic providers to perform oral health screenings as a routine part of care for all eligible patients during the study period. Clinicians entered screening results both in the patient's electronic health record and on the visit Superbill. ${ }^{35}$ Research staff sent screening reminders and oral health review guides to the clinic providers twice during the study.

\section{Patient Self-Report Dental Access Survey}

Clinic front-desk staff distributed copies of the dental access survey to all eligible patients when they checked in for their appointment. Staff members encouraged parents to assist their children with the survey, and consent/assent was implied when a completed survey was returned. This survey consisted of 12 questions that were designed to assess patient insurance status, oral health care patterns, and self-reported unmet dental needs. We selected questions through consultation with the local dental champion, providers at the primary care facility, and personnel from the medical and oral health PBRNs. When possible we modeled questions after existing health assessment surveys. ${ }^{9,10}$ Patients completed the surveys in the waiting room or encounter room and returned them to the clinic check-out desk.

\section{Statistical Analysis}

Two authors (TH and MD) reviewed the free text of patient self-reported unmet dental needs and developed 12 unique condition categories. Three authors (TH, MD, and SB) coded the original text into the relevant categories. A patient's unmet dental needs could fall into multiple categories (eg, unmet needs regarding both caries/fillings/crowns and extractions).

We performed all statistical analyses using SPSS for Windows (version 17.0, SPSS, Inc., Chicago, IL). In the case of duplicate surveys, we used the first completed. $\chi^{2}$ goodness-of-fit tests were used to compare age and sex distributions of study participants with the age and sex distributions of all eligible patients who presented to the clinic during the study period. We used Pearson $\chi^{2}$ tests (or fisher's exact test, for $2 \times 2$ cross-tabulation tables) to compare the distribution of oral health conditions and unmet needs across age and sex categories.

\section{Results \\ Patient Sample}

During the study period, 1655 unique patients met the study eligibility criteria (Table 1). Of these patients, $40.7 \%$ (674) received the oral health screening and $66.9 \%$ (1108) completed the dental access survey. More than one third of the patients ( $\mathrm{n}=591,35.7 \%)$ both completed the survey and received the screening. Performance of the oral health screen varied among the individual clinic providers, with screening rates ranging from $7.7 \%$ to $66.8 \%$ (mean $38.6 \%$, SD, $19.6 \%$ ).

As compared with all patients who presented for care, there were no statistically significant differences in the sex or age composition of patients who received the oral health screening $(33.7 \%$ male, $P=.21$; by age, $P=.74)$ or who completed the patient survey $(35.6 \%$ male, $P=.73$; by age, $P=$ .09) (Table 1). Furthermore, the age by sex distribution of patients who completed the survey or who received the screening were not significantly different from all eligible patients presenting for care during the study period (all $\chi^{2}$ goodness-of-fit $P$ values were non-significant).

\section{Oral Health Screening Condition Detection and Distribution}

For the 674 unique patients who received an oral health screening, $46.0 \%(n=310)$ had oral health conditions; a total of 394 conditions were detected (Table 2). Of these 310 patients, $76.8 \%$ had only 
Table 1. Demographic Characteristics of Eligible Patients and Patients Who Received the Oral Health Screening, Dental Access Survey, or both Screening and Survey during the 6-Week Study Period*

\begin{tabular}{|c|c|c|c|c|}
\hline Characteristic & $\begin{array}{l}\text { Eligible Patients }{ }^{\dagger} \\
\text { (n [\%]) }\end{array}$ & $\begin{array}{l}\text { Patients Completing } \\
\text { Survey }^{\ddagger}(\mathrm{n}[\%])\end{array}$ & $\begin{array}{c}\text { Patients Who Received } \\
\text { Oral Screening } \$ \\
\text { (n [\%]) }\end{array}$ & $\begin{array}{l}\text { Patients Who Completed the } \\
\text { Survey and Who Received an } \\
\text { Oral Screeningll (n [\%]) }\end{array}$ \\
\hline Total patients & $1655(100)$ & $1108(66.9)$ & $674(40.7)$ & $591(35.7)$ \\
\hline \multicolumn{5}{|l|}{ Sex } \\
\hline Male & $597(36.1)$ & $394(35.6)$ & $227(33.7)$ & $202(34.2)$ \\
\hline Female & $1058(63.9)$ & $714(64.4)$ & $447(66.3)$ & $389(65.8)$ \\
\hline \multicolumn{5}{|l|}{ Age (years) } \\
\hline $1-4$ & $117(7.1)$ & $59(5.3)$ & $42(6.2)$ & $33(5.6)$ \\
\hline $5-14$ & $142(8.6)$ & $90(8.1)$ & $56(8.3)$ & $52(8.8)$ \\
\hline $15-44$ & $503(30.4)$ & $333(30.1)$ & $213(31.6)$ & 187 (31.6) \\
\hline $45-64$ & $485(29.3)$ & $328(29.6)$ & $189(28.0)$ & $166(28.1)$ \\
\hline$\geq 65$ & $408(24.7)$ & $298(26.9)$ & $174(25.8)$ & $153(25.9)$ \\
\hline
\end{tabular}

*Because of rounding, some percentage grouping totals do not equal $100 \%$.

${ }^{+}$Unique patients older than 12 months of age out of the 2316 total patient visits during the 6-week study period.

${ }^{\ddagger} \chi^{2}$ goodness-of-fit $P=.730$ for sex; $P=.093$ for age.

${ }^{\$} \chi^{2}$ goodness-of-fit $P=.209$ for sex; $P=.741$ for age.

$\| \chi^{2}$ goodness-of-fit $P=.356$ for sex; $P=.642$ for age.

one oral health condition, $18.4 \%$ had 2 conditions, and $4.8 \%$ had 3 conditions. Only one patient had 4 oral health conditions. Commonly detected conditions included partial edentulism (24.5\%), dental caries $(12.9 \%)$, complete edentulism $(9.9 \%)$, and cracked/broken teeth $(8.9 \%)$.

There were significant differences in the distribution of certain oral health conditions (caries, edentulism, partial edentulism, cracked teeth) by patient age (all $\chi^{2}$ test $P<.05$ ) (Table 2). Patients older than 65 were the most likely to have complete edentulism $(29.9 \%)$ or partial edentulism $(39.7 \%)$. Dental caries were most prevalent in younger patients aged 5 to 14 years of age $(16.1 \%)$ and in young adult/middle-aged patients aged 15 to 44 years of age $(18.8 \%)$, whereas broken or cracked

Table 2. Outcomes of Oral Health Clinical Screening by Age Category $(n=674)$

\begin{tabular}{|c|c|c|c|c|c|c|c|}
\hline \multirow[b]{2}{*}{ Outcomes (ICD-9-CM Diagnosis Codes) } & \multirow[b]{2}{*}{$\begin{array}{c}\text { All } \\
\text { Patients } \\
\text { (n [\%] })\end{array}$} & \multicolumn{6}{|c|}{ Age (Years) } \\
\hline & & $\begin{array}{c}1-4 \\
(\mathrm{n}[\%])\end{array}$ & $\begin{array}{c}5-14 \\
(\mathrm{n}[\%])\end{array}$ & $\begin{array}{c}15-44 \\
(\mathrm{n}[\%])\end{array}$ & $\begin{array}{c}45-64 \\
(\mathrm{n}[\%])\end{array}$ & $\begin{array}{c}\geq 65 \\
(\mathrm{n}[\%])\end{array}$ & $P$ \\
\hline Patients who received screening* & $674(100.0)$ & $42(6.2)$ & $56(8.3)$ & $213(31.6)$ & $189(28.0)$ & $174(25.8)$ & \\
\hline No condition(s) detected (V70.7) & $364(54.0)$ & $36(9.9)$ & $45(12.4)$ & $153(42.0)$ & $90(24.7)$ & $40(11.0)$ & $<.001$ \\
\hline Oral health condition(s) detected & $310(46.0)$ & $6(1.9)$ & $11(3.6)$ & $60(19.4)$ & $99(31.9)$ & $134(43.2)$ & \\
\hline \multicolumn{8}{|c|}{ Distribution of detected oral health condition $(s)^{\dagger}$} \\
\hline Partial edentulism (525.50) & $165(24.5)$ & $1(2.4)$ & $1(1.8)$ & $26(12.2)$ & $68(36.0)$ & $69(39.7)$ & $<.001$ \\
\hline Caries (521.00) & $87(12.9)$ & $3(7.1)$ & $9(16.1)$ & $40(18.8)$ & $23(12.2)$ & $12(6.9)$ & .007 \\
\hline Complete edentulism (525.40) & $67(9.9)$ & $0(0.0)$ & $0(0.0)$ & $2(0.9)$ & $13(6.9)$ & $52(29.9)$ & $<.001$ \\
\hline Broken/cracked teeth (521.81) & $60(8.9)$ & $0(0.0)$ & $1(1.8)$ & $20(9.4)$ & $22(11.6)$ & $17(9.8)$ & .048 \\
\hline Unspecified disorder (525.9) & $10(1.5)$ & $2(4.8)$ & $1(1.8)$ & $2(0.9)$ & $1(0.5)$ & $4(2.3)$ & .238 \\
\hline Abscess (522.5) & $5(0.7)$ & $1(2.4)$ & $0(0.0)$ & $1(0.5)$ & $1(0.5)$ & $2(1.1)$ & .614 \\
\hline
\end{tabular}

*Percentages in the first 3 rows are based on the number of patients in the age category divided by the number in the "All Patients" column.

${ }^{\dagger}$ For the subcategories of detected oral health condition(s), percentages are based on the number of patients who received the screening within each age category (ie, caries in the $1-4$ years old category: $3 / 42=7.1 \%$ ). Note that some patients had more than one condition detected.

ICD-9-CM, International Statistical Classification of Diseases and Related Health Problems 9-Clinical Modification. 
teeth were most common for the 15 - to 44 -year and 45 - to 64-year age groups $(9.4 \%$ and $11.6 \%$, respectively). Generally there were no statistically significant differences in the distribution of oral health conditions by sex. However, male patients older than age 65 were significantly more likely than their female counterparts to have caries present $(P=.01)$.

\section{Self-Reported Insurance, Dental Visits, Health Status, and Unmet Dental Needs}

Most patients reported having health insurance $(91.2 \%, \mathrm{n}=1006)$, but only $56.5 \%$ had both health and dental insurance (Table 3). Fewer than $1 \%$ of the patients $(n=3)$ reported having dental insurance in the absence of health insurance. Although $66.0 \%$ of the patients reported having a source of regular dental care, only $53.8 \%$ had seen a dentist during the past year. Patients with dental insurance relative to no insurance were 6 times more likely to report that they had a regular source of dental care (Table 4). This was significantly different from patients who had only health insurance; these patients were twice as likely to report having a regular source of dental care when compared with uninsured patients $(P \leq .002)$.

Patient report of overall health was significantly correlated with report of oral health (Spearman's rho $=0.59 ; P<.001)$. Most respondents $(70.6 \%)$ reported "average" or "good" oral health. Among those who reported poor oral health, $38.2 \%$ reported poor overall health and only $1.6 \%$ reported excellent overall health. Patients with dental insurance were 3 times as likely to report that their oral health was "good" or "excellent" and twice as likely to report the same about their overall health when compared with those without any insurance. $\mathrm{Pa}$ tients with dental insurance were more likely to report having no unmet dental needs than uninsured patients (odds ratio [OR], 3.1; 95\% CI, 2.04.9). This was significantly different $(P \leq .002)$ from patients with health insurance only, who were also more likely to report no unmet dental needs (OR, 2.0; 95\% CI, 1.3-3.1).

\section{Perceived Unmet Dental Needs: Results, Risk Factors, and Screening Correspondence}

More than one fourth of survey respondents $(28.0 \%)$ indicated that they had unmet dental needs (Table 3). The most commonly reported needs included cavities/crowns (11.2\%), dentures (6.4\%),
Table 3. Results from the Patient Survey $(\mathrm{n}=\mathbf{1 1 0 8})$

\begin{tabular}{lc}
\hline Variables & $\mathrm{n}(\%)$ \\
\hline Insurance & \\
Health (yes) & $1006(91.2)$ \\
Dental (yes) & $571(51.9)$ \\
Health and dental (yes) & $567(56.5)$ \\
Source of regular dental care (yes) & $717(66.0)$ \\
Tried to see dentist during the past year & $654(59.5)$ \\
Had a dental visit during the past year & $596(53.8)$ \\
Self-rated oral health & \\
Poor & $128(11.7)$ \\
Average & $345(31.5)$ \\
Good & $428(39.1)$ \\
Excellent & $194(17.7)$ \\
Self-rated overall health & \\
Poor & $80(7.4)$ \\
Average & $323(29.7)$ \\
Good & $530(48.7)$ \\
Excellent & $155(14.2)$ \\
Unmet dental need (yes) & $304(28.0)$ \\
Stated unmet dental needs by condition* & \\
Cavities/fillings/crowns & $124(11.2)$ \\
Dentures & $71(6.4)$ \\
Exam/cleaning & $54(4.9)$ \\
Bxtractions & $44(4.0)$ \\
Lack of money/needs insurance & $28(2.5)$ \\
Other coded conditions & \\
Other miscellaneous & \\
\hline & $37(3.3)$ \\
& $52(4.7)$ \\
& $34(3.1)$ \\
\hline
\end{tabular}

*Total stated unmet dental need categories do not add to 304 unique patients because survey respondents could self-report multiple unmet needs.

${ }^{\dagger}$ Other coded conditions included periodontics ( $\left.\mathrm{n}=15,1.4 \%\right)$; endodontics ( $\mathrm{n}=11,1.0 \%$ ); orthodontics ( $\mathrm{n}=11,1.0 \%$ ); bridges $(\mathrm{n}=11,1.0 \%)$; and general statements such as "a lot" $(\mathrm{n}=5,0.5 \%)$. One patient reported multiple other coded unmet needs, thus individual values do not equal the table total $(\mathrm{n}=52$, 4.7\%).

${ }^{\ddagger}$ Other miscellaneous included dental needs stated by patients that were not captured in the 11 coded categories. Most patients in this category also specified one of the coded unmet needs.

examination/cleaning (4.9\%), extractions (4.0\%), lack of money to obtain care (3.3\%), and broken/ cracked teeth $(2.5 \%)$. Other coded categories of unmet dental needs were identified by $4.7 \%$ of the respondents and included references to periodontics, endodontics, orthodontics, bridges, or multiple needs (implied with responses such as "a lot"). Other miscellaneous needs were identified by $3.1 \%$ of the respondents.

Patients who saw a dentist during the past year were 10.4 times more likely to report that they had no unmet dental needs (95\% CI, 2.9-42). Patients 
Table 4. Association of Insurance Status with Perceived Dental Needs and Health Outcomes $(n=1097) *$

\begin{tabular}{|c|c|c|c|c|}
\hline \multirow[b]{2}{*}{ Dental/Health Outcome } & \multicolumn{2}{|c|}{$\begin{array}{l}\text { Dental Insurance }{ }^{\dagger} \text { Relative to } \\
\text { No Insurance } \S\end{array}$} & \multicolumn{2}{|c|}{$\begin{array}{l}\text { Health Insurance Only }{ }^{\ddagger} \\
\text { Relative to No Insurance }\end{array}$} \\
\hline & OR $(95 \% \mathrm{CI})$ & $P$ & OR $(95 \% \mathrm{CI})$ & $P$ \\
\hline Had source of regular dental care & $6.4(4.0-10.3)^{\|}$ & $<.001$ & $1.9(1.2-3.0)$ & .008 \\
\hline Tried to see dentist during the past year & $3.8(2.4-6.0)^{\|}$ & $<.001$ & $1.9(1.2-3.0)$ & .006 \\
\hline Had dental visit during the past year & $2.9(1.0-8.2)$ & .054 & $2.9(1.0-0.9)$ & .061 \\
\hline Oral health rated good/excellent & $2.8(1.8-4.5)^{\|}$ & $<.001$ & $1.7(1.1-2.7)$ & .024 \\
\hline Overall health rated good/excellent & $1.9(1.2-3.0)^{\|}$ & $<.001$ & $1.1(0.7-1.7)$ & .82 \\
\hline No stated unmet dental need(s) & $3.1(2.0-4.9)^{\|}$ & $<.001$ & $2.0(1.3-3.1)$ & .004 \\
\hline
\end{tabular}

*Odds ratio (OR) estimates were limited to patients with information about both dental and health insurance status.

${ }^{+} 99.5 \%$ of patients $(\mathrm{n}=567$ of 570$)$ with dental insurance also carried health insurance.

$\neq 43.3 \%$ of patients $(n=433$ of 1000$)$ with health insurance did not carry dental insurance.

$\$ 8.5 \%$ of patients $(\mathrm{n}=94)$ carried no insurance.

"Differences between dental insurance and health insurance significant at $P \leq .002$.

who had a regular source of dental care were more than 5 times as likely to report having no unmet dental needs (OR, 5.6; 95\% CI, 4.2-7.4). Patients without unmet dental needs were more likely to rate their oral health or overall health as good or excellent when compared with those who reported having unmet needs (OR, 5.6; 95\% CI, 4.2-7.5 and OR, 3.4; 95\% CI, 2.6-4.5, respectively).

There were no significant differences in report of unmet dental needs by sex. The reported types of unmet dental needs differed significantly across age categories. Patients 5 to 44 years of age reported needing cavities repaired and extractions at significantly higher rates than patients in the other age categories. Older patients reported dentures as an unmet need 6 times more often than younger patients. Of the 591 patients $(35.7 \%)$ who both completed the survey and received the screening examination, those who screened positive for any oral health condition were more likely to report unmet dental needs on the survey than those who had no conditions detected (OR, 3.6; 95\% CI, 2.4-5.2).

\section{Discussion}

Our findings indicate that oral health conditions are common in patients who present for regularly scheduled visits in rural primary care; approximately half of the screened patients experience an oral health condition and almost one-third report unmet dental needs. Results from the patient survey indicated that a lack of dental insurance was associated with greater reported unmet dental needs and lower levels of self-rated oral and overall health. For most oral health outcomes the presence of dental insurance was significantly more protective than health insurance only. Although there were no statistically significant variations in the distribution of oral health conditions by sex, the prevalence of oral health conditions varied significantly by patient age on both the screening and survey in predicted ways. Specifically, partial edentulism and complete edentulism were more prevalent among patients older than age 65 whereas cavities were most common for patients aged 5 to 44 years.

Few studies have explored the prevalence of oral health conditions and unmet dental needs as they are presented in a primary care practice in patients with ages spanning across the entire lifespan. Our results describe an important health issue in primary care but do have some limitations. There was substantial variability in the rate at which providers conducted oral health screenings. Providers and clinic staff believed this was a result of differential study interest, variations in workload, and competing priorities during the office visit. In addition, $33 \%$ of patients who presented for care did not complete the dental access survey. Clinic staff reported that all patients who presented for a scheduled appointment received the survey, but missing surveys resulted when patients declined because they felt too sick, were not interested, had privacy concerns, or were late for an appointment and therefore lacked the time. A small number of patients were unable to complete the survey because of cognitive impairment or literacy issues. $\mathrm{Al}$ though it is possible that patients who had oral health concerns were more likely to receive the 
screening or complete the survey, we believe such a bias was small because both were implemented as aspects of routine care. There were no significant differences in sex, age, or age by sex comparisons between all eligible patients who presented for care during the study period and those who completed the study activities. Thus, the sample seems representative of the clinic population.

It is likely that both the detection of oral health conditions and patients self-report of unmet needs were underestimated in this study. The primary care clinicians received abbreviated training about oral health assessments; lacked access to normal diagnostics such as dental radiographs and magnification; and were in environments that lacked a dental chair, high-intensity lighting, and designated screening time, thereby limiting diagnostic abilities. In addition, low dental literacy among rural populations may lead to underreporting of dental needs because of differences in what conditions are perceived to require care. ${ }^{12,13}$

In this study $46.2 \%$ of patients reported that they had not seen a dentist during the past year, a rate $11.5 \%$ lower than the proportion for rural residents in national studies. ${ }^{13} \mathrm{We}$ performed screenings and surveys in a primary care setting and our data may not be representative of national patterns because patients who present to primary care may be different from the general population. Our participants who had dental insurance or health insurance only were almost 3 times as likely to report having a dental visit during the past year when compared with patients who did not have insurance, although this finding was only marginally significant.

The conditions that impede access to dental care are multifactorial and include social, economic, and cultural factors. ${ }^{12,34,36}$ Rural populations are particularly at risk because they tend to be less affluent, older, and less likely to have private insurance than their urban counterparts. ${ }^{13,37}$ A paucity of dental providers and/or providers who accept low-income patients further impedes access for rural populations. $^{12,13}$

The importance of access to dental care is heightened by the increasingly recognized connections between oral and systemic health. Several studies have identified the potential for pediatricians and family medicine providers to improve oral health and reach patients who are unlikely to make dental visits. ${ }^{23,24,27}$ Barriers that inhibit greater involvement in dental care include medical providers' limited knowledge and difficulty with referring subgroups that require treatment for oral care. ${ }^{24}$ These factors, plus competing demands for preventive health and chronic illness care, are important hurdles to expanding the scope of care.

Despite these barriers, pediatricians agree that oral health assessments and preventive counseling should be a routine part of well-child care. ${ }^{24}$ Family physicians in our study echoed the desire to provide assessment, preventive counseling, and referrals for oral health. In a follow-up survey, participating providers noted that, although completing the oral health screening was more time consuming than they had anticipated, they appreciated the opportunity to quantify their patients' unmet dental needs. Many commented that this study helped them realize how often oral health is overlooked in primary care and that the training improved their ability to conduct oral health exams. One clinician commented, "I realized I looked past the teeth to the pharynx in my normal oral examination." Study clinicians indicated that it was important for primary care providers to be able to identify dental conditions, reinforce the importance of regular dental care to their patients, and serve as a referral point for patients who had oral health needs.

Family physicians provide care for one third of children in the US population, ${ }^{38}$ and a growing number of studies use this setting as an opportunity to provide pediatric preventive dental services, including risk assessment, screening, referral, application of fluoride varnish, and oral health education. ${ }^{28,30,39}$ Other studies address the dental needs of geriatric patients in primary medical care settings. ${ }^{39}$ Our data indicate that attention to oral health in primary care should not be limited to pediatric or geriatric patients because conditions appear among patients across all ages.

\section{Conclusion}

Additional research is needed to assess the prevalence of oral health conditions and unmet needs in other care settings. Our results offer a picture of oral health conditions and unmet dental needs experienced by patients in one rural primary care clinic. Further, our data corroborate the results of an objective oral health screen with a self-report survey. These findings contribute to the literature on oral health disparities, provide an important 
foundation for local efforts to address the community's unmet dental needs, and may inform future interventions to address oral health in primary care settings.

If medical providers are to confidently address oral health across the lifespan in patients presenting in the primary care setting, attention to training, adequate referral opportunities, and reimbursement are necessary. ${ }^{24,40}$ Policymakers must also acknowledge the important links between oral and systemic health. ${ }^{41}$ Partnerships among primary care settings, PBRNs, and community health partners may provide opportunities to develop and implement interventions to reduce the burden of oral health disease and facilitate overall patient health and well-being.

\footnotetext{
The authors would like to thank the members of the Baker County Community Health Improvement Partnership for their assistance with all stages of project development, including problem identification, study design, data analysis, and project next steps. Special appreciation is given to Cindy Denne, Eva Sorensen, and the staff, providers, and patients at Eastern Oregon Medical Associations-their dedication to improving community health made this project possible. Thanks to Cindy Barnes and Emilia Adams for data entry assistance. Richard Deyo, MD, MPH; Lisa Lyman, PhD; Jean O'Malley, MPH; and members of the OHSU Department of Family Medicine Working on Research Collaboratively Group provided helpful feedback about the manuscript.
}

\section{References}

1. US Department of Health and Human Services. Oral health in America: a report of the Surgeon General. Rockville, MD: US Department of Health and Human Services, National Institute of Dental and Craniofacial Research; 2000.

2. Hollister MC, Weintraub JA. The association of oral status with systemic health, quality of life, and economic productivity. J Dent Educ 1993;57:901-12.

3. US Department of Health and Human Services. Healthy People 2010: understanding and improving health. Washington, DC; US Department of Health and Human Services; 2000.

4. Fowler EB, Breault LG, Cuenin MF. Periodontal disease and its association with systemic disease. Mil Med 2001;166:85-9.

5. Williams RC. Understanding and managing periodontal diseases: a notable past, a promising future. J Periodontol 2008;79(8 Suppl):1552-9.

6. Mealey BL, Rose LF. Diabetes mellitus and inflammatory periodontal diseases. Compend Contin Educ Dent 2008;29:402-8.

7. Nelson WE, Behrman RE, Kliegman RM, Marvin AM, editors. Textbook of pediatrics. 15th edition. Philadelphia, PA: WB Saunders; 1996.
8. Edelstein BL. Evidence-based dental care for children and the age 1 dental visit. Pediatr Ann 1998;27: 569-74.

9. Mueller CD, Schur CL, Paramore LC. Access to dental care in the United States: estimates from a 1994 survey. J Am Dent Assoc 1998;129:429-37.

10. Newacheck PW, Hughes DC, Hung Y-Y, Wong S, Stoddard JJ. The unmet health needs of America's children. Pediatrics 2000;105:989-97.

11. Centers for Disease Control and Prevention. Dental service use and dental insurance coverage-United States, Behavioral Risk Factor Surveillance System, 1995. MMWR Morb Mortal Wkly Rep 1997;46: 1199-203.

12. Allison RA, Manski RJ. The supply of dentists and access to care in rural Kansas. J Rural Health 2007; 23:198-206.

13. Vargas CM, Dye BA, Hayes KL. Oral health care utilization by US rural residents. National Health Interview Survey 1999. J Public Health Dent 2003; 63:150-7.

14. Vargas CM, Dye BA, Hayes KL. Oral health status of rural adults in the United States. J Am Dent Assoc 2002;133:1672-81.

15. Vargas CM, Ronzio CR, Hayes KL. Oral health status of children and adolescents by rural residence. United States. J Rural Health 2003;19:260-8.

16. Vargas CM, Yellowitz JA, Hayes KL. Oral health status of older rural adults in the United States. J Am Dent Assoc 2003;134:479-86.

17. Martin AB, Wang E, Probst JC, Hale N, Johnson AO. Dental health and access to care among rural children: a national and state portrait. Columbia, SC: South Carolina Rural Health Research Center; 2008.

18. Sheiham A, Watt RG. The common risk factor approach: a rational basis for promoting oral health. Community Dent Oral Epidemiol 2000;28:399-406.

19. Mouradian WE, Wehr E, Crall JJ. Disparities in children's oral health and access to dental care. JAMA 2000;284:2625-31.

20. Evans CA, Kleinman DV. The Surgeon General's Report on America's oral health: opportunities for the dental profession. J Am Dent Assoc 2000;131: $1721-8$.

21. US Department of Health and Human Services. National call to action to promote oral health: a public-private partnership under the leadership of The Office of the Surgeon General. Report no. 035305. Rockville, MD: US Department of Health and Human Services, Public Health Services, National Institutes of Health, National Institute of Dental and Craniofacial Research; 2003.

22. Mouradian WE, Berg JH, Somerman MJ. Addressing disparities through dental-medical collaborations, part 1. The role of cultural competency in health disparities: training of primary care medical practitioners in children's oral health. J Dent Educ. 67(8):860-8, 2003 Aug 1. 
23. dela Cruz GG, Rozier RG, Slade GD. Dental screening and referral of young children by pediatric primary care providers. Pediatrics 2004;114:e64252.

24. Lewis CW, Grossman DC, Domoto PD, Deyo RA. The role of the pediatrician in the oral health of children: a national survey. Pediatrics 2000;106:E84.

25. Fagnan LJ, Morris C, Shipman SA, Holub J, King A, Angier H. Characterizing a practice-based research network: Oregon Rural Practice-based Research Network (ORPRN) survey tools. J Am Board Fam Med 2007;20:204-19.

26. McGinnis P, Przybilla J. Community Health Improvement Partnership: a rural community health development process. Available at http://www.ohsu. edu/research/orprn/resources/community.html. Accessed 8 June 2010.

27. Mouradian WE, Schaad DC, Kim S, et al. Addressing disparities in children's oral health: a dentalmedical partnership to train family practice residents. J Dent Educ 2003;67:886-95.

28. Rozier RG, Sutton BK, Bawden JW, Haupt K, Slade GD, King RS. Prevention of early childhood caries in North Carolina medical practices: implications for research and practice. J Dent Educ 2003;67:876-85.

29. Slade GD, Rozier RG, Zeldin LP, Margolis PA. Training pediatric health care providers in prevention of dental decay: results from a randomized controlled trial. BMC Health Serv Res 2007;7:176.

30. Wawrzyniak MN, Boulter S, Giotopoulos C, Zivitksi J. Incorporating caries prevention into the well-child visit in a family medicine residency. Fam Med 2006;38:90-2.

31. Oregon Office of Rural Health. Baker City Service Area Profile, 2008. Portland, OR: Office of Rural Health; 2009.
32. Oregon Office of Rural Health. Office of Rural Health primary care profiles. Portland, OR: Office of Rural Health; 2007.

33. Lewis C, Lynch H, Johnston B. Dental complaints in emergency departments: a national perspective. Ann Emerg Med 2003;42:93-9.

34. Lukes SM, Simon B. Dental decay in southern Illinois migrant and seasonal farmworkers: an analysis of clinical data. J Rural Health 2005;21:254-8.

35. Backer LA. In search of a superbill. Fam Prac Manag 2006; $13: 43-6$.

36. Guay AH. Access to dental care: solving the problem for underserved populations. J Am Dent Assoc 2004; 135:1599-605.

37. Casey MM, Davidson G, Moscovice I, Born D. Access to dental care for rural low income and minority populations. Report No. 54. Minneapolis, MN: University of Minnesota, Center RHR; 2004.

38. Phillips RL Jr, Bazemore AW, Dodoo MS, Shipman SA, Green LA. Family physicians in the child health care workforce: opportunities for collaboration in improving the health of children. Pediatrics 2006; 118:1200-6.

39. Lowe C, Blinkhorn AS, Worthington HV, Craven R. Testing the effect of including oral health in general health checks for elderly patients in a medical practice-a randomized controlled trial. Community Dent Oral Epidemiol 2007;35:12-7.

40. Casamassimo PS. Oral health in primary care medicine: practice and policy challenges. Am Fam Physician 2004;70(11):2074, 2076.

41. Fisher-Owens SA, Barker JC, Adams S, et al. Giving policy some teeth: routes to reducing disparities in oral health. Health Aff 2008;27:404-12. 